\title{
Social modulation of brain monoamine levels in zebrafish
}

\author{
Magda C. Teles ${ }^{a, b}$, S. Josefin Dahlbom ${ }^{c}$, Svante Winberg ${ }^{c}$, Rui F. Oliveira ${ }^{a, b, *}$ \\ a ISPA-Instituto Universitário, Unidade de Investigação em Eco-Etologia, Rua Jardim do Tabaco 34, 1149-041, Lisboa, Portugal \\ ${ }^{\mathrm{b}}$ Champalimaud Neuroscience Programme, Instituto Gulbenkian de Ciência, Rua da Quinta Grande 6, 2780-156, Oeiras, Portugal

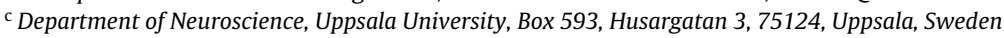

\section{H I G H L I G H T S}

- Zebrafish were exposed to different fighting experiences: winning, losing and mirror-fighting.

- Winners show higher serotonergic and dopaminergic activity in the telencephalon.

- Losers show higher serotonergic in the optic tectum.

- No significant changes in monoamine activity were observed in mirror fighters.

- Monoamines are differentially regulated by social interactions in different brain regions.

\section{A R T I C L E I N F O}

\section{Article history:}

Received 21 May 2013

Received in revised form 27 June 2013

Accepted 1 July 2013

Available online 11 July 2013

\section{Keywords:}

Aggressive behaviour

Behavioural plasticity

Neuromodulators

Serotonin

Dopamine

Zebrafish

\begin{abstract}
A B S T R A C T
In social species animals tend to adjust their social behaviour according to the available social information in the group, in order to optimize and improve their one social status. This changing environment requires for rapid and transient behavioural changes that relies primarily on biochemical switching of existing neural networks. Monoamines and neuropeptides are the two major candidates to mediate these changes in brain states underlying socially behavioural flexibility. In the current study we used zebrafish (Danio rerio) males to study the effects of acute social interactions on rapid regional changes in brain levels of monoamines (serotonin and dopamine). A behavioural paradigm under which male zebrafish consistently express fighting behaviour was used to investigate the effects of different social experiences: winning the interaction, losing the interaction, or fighting an unsolved interaction (mirror image). We found that serotonergic activity is significantly higher in the telencephalon of winners and in the optic tectum of losers, and no significant changes were observed in mirror fighters suggesting that serotonergic activity is differentially regulated in different brain regions by social interactions. Dopaminergic activity it was also significantly higher in the telencephalon of winners which may be representative of social reward. Together our data suggests that acute social interactions elicit rapid and differential changes in serotonergic and dopaminergic activity across different brain regions.
\end{abstract}

(c) 2013 Elsevier B.V. All rights reserved.

\section{Introduction}

In order to optimize the benefits of group living and to minimize its costs, social animals need to adjust the expression of their social behaviour according to daily changes in their social environment. This ability of an individual to optimize its social behaviour depending on available social information (aka social competence, [1]), depends primarily on mechanisms that allow for rapid and transient behavioural changes. Given the speed and liability of this type of behavioural flexibility, such mechanisms are

\footnotetext{
* Corresponding author at: ISPA-Instituto Universitário, Unidade de Investigação em Eco-Etologia, Rua Jardim do Tabaco 34, 1149-041, Lisboa, Portugal. Fax: +351218860954

E-mail address: ruiol@ispa.pt (R.F. Oliveira).
}

expected to rely on socially driven biochemical switching of existing neural networks, rather than on structural rewiring of neural circuits [2]. In recent years evidence accumulated showing how neuromodulators can change the activity and even the connectivity of neural circuits in a way that each structural circuit, as represented by its connectome, may include multiple functional circuits, with some of them active and some others latent at a given moment in time [3]. Different neuromodulatory agents may interact with specific circuits and alter their functional properties, promoting either excitatory or inhibitory states. Monoamines and neuropeptides are considered the two major classes of neuromodulators, and the action of both on social behaviour as well as their sensitivity to environmental factors, have been extensively documented [4,5], which makes them major candidates to mediate changes in brain states underlying socially driven behavioural flexibility. 
Monoamines have been implicated in the regulation of motivated behaviours and among them the role of the serotonergic system on the control of aggressive motivation has been demonstrated both in vertebrate and in invertebrate species [6,7]. Interestingly the effects of serotonin (5-hydroxytryptamine, 5-HT) on aggressive behaviour are to some extent paradoxical. While several studies have pointed out that pharmacological manipulations that increase 5-HT inhibit aggression in a wide range of vertebrates, from fish to humans [8], other studies, in contrast, have showed increased serotonergic activity in specific brain regions during the expression of aggressive behaviour [8-10]. Moreover, the $5-\mathrm{HT}_{1 \mathrm{~A}}$ and $5-\mathrm{HT}_{1 \mathrm{~B}}$ receptors exert functionally opposing roles in various behavioural and physiological processes such as appetite, sexual libido, motor activity, and thus it is reasonable to consider that this divergence may also be present in aggressive behaviour [11-13]. Therefore, the role of 5-HT on the regulation of social behaviour cannot be put simply in terms of pure inhibition or pure facilitation of aggression, but rather as a function of environmental context. The effects of dopamine (DA) on aggression are also paradoxical. For example in mammals, while D1 and D2 dopamine receptor antagonists reduce aggression [14], D2 receptors in the medial preoptic area ( $\mathrm{mPOA}$ ) and anterior hypothalamus facilitate affective defense behaviour [15]. On the other hand, the mesocorticolimbic dopamine system has been shown to be involved in the preparation and execution of aggressive acts [16-20]. These neurochemical studies link elevated dopamine and its metabolites in prefrontal cortex and nucleus accumbens not only to the initiation of attacks and threats, but also to defensive and submissive responses in reaction of being attacked [19,21]. The transition between behavioural states (e.g. inhibition or promotion of aggressive behaviours) in both monoaminergic systems appears to be sensitivity to different social contexts, which make these neuromodulators tremendously important in the regulation of social interactions.

The high diversity and plasticity of social behaviour among teleost fish makes them excellent models for comparative studies on the mechanisms of social plasticity [22]. In many fish species social systems are characterized by reversible dominance hierarchies, where animals have to adjust the expression of their social behaviour to their perceived social status. In these social systems rapid changes in behavioural output occur, driven by the assessment that the animal does of the social interactions in which it is involved.

In this paper we used zebrafish (Danio rerio) males to study the effects of acute social interactions on rapid regional changes in brain levels of monoamines. Zebrafish were chose as a model species given their increasing use in behavioural neuroscience research and their flexible social behaviour. Zebrafish is a groupliving species that in nature form shoals [23] but when allowed to interact in pairs, form dominance hierarchies [24]. In this species aggression is commonly used by dominant individuals to get access to spawning sites and to protect their social status from competitors [25]. Recently, our group developed a behavioural paradigm under which male zebrafish consistently express fighting behaviour and characterized the structure of these fights in male dyads [26]. Here the same paradigm is used to investigate the effects of different social experiences (i.e. individuals experiencing a victory, a defeat or fighting an unsolved interaction) on serotonin and dopamine levels in different brain regions.

\section{Materials and methods}

\subsection{Animals and housing}

All subjects used in this experiment were adult wild-type $(A B)$ zebrafish breed and held at Instituto Gulbenkian de Ciência (IGC, Oeiras, Portugal). Fish were kept in a recirculating system (ZebraTec, 93 Tecniplast), at $28^{\circ} \mathrm{C}$ with a $14 \mathrm{~L}: 10 \mathrm{D}$ photoperiod. Water system was monitored for nitrites $(<0.2 \mathrm{ppm})$, nitrates $(<50 \mathrm{ppm})$ and ammonia (0.01-0.1 ppm), while $\mathrm{pH}$ and conductivity were maintained at 7 and $700 \mu \mathrm{Sm}$ respectively. Fish were fed twice a day with commercial food flakes in the morning and Artemia salina in the afternoon, except on the day of the experiments.

\subsection{Experimental design}

In the present study a behavioural paradigm previously developed for the study of zebrafish aggressive behaviour was used [26]. Thirty-two adult males ( 8 in each experimental treatment) matched for standard length (mean $\pm \mathrm{SEM}$ : $2.81 \pm 0.026 \mathrm{~cm}$ ) and body mass (mean \pm SEM: $0.350 \pm 0.009 \mathrm{~g}$ ) were grouped in dyads. There were three types of dyads: (1) real opponent fight: the fish fought with a conspecific; (2) mirror fight: the fish fought with their own mirror image; (3) no fight: the fish had no agonistic interaction (Fig. 1). From these three types of dyads, came out four experimental conditions: winning the interaction, losing the interaction, fighting an unsolved interaction, or experience no interaction (control group). Subjects were always tested in pairs, in order to give them access to conspecific odours, which would otherwise only be present in real opponent dyads, therefore avoiding confounding effects of putative chemical cues.
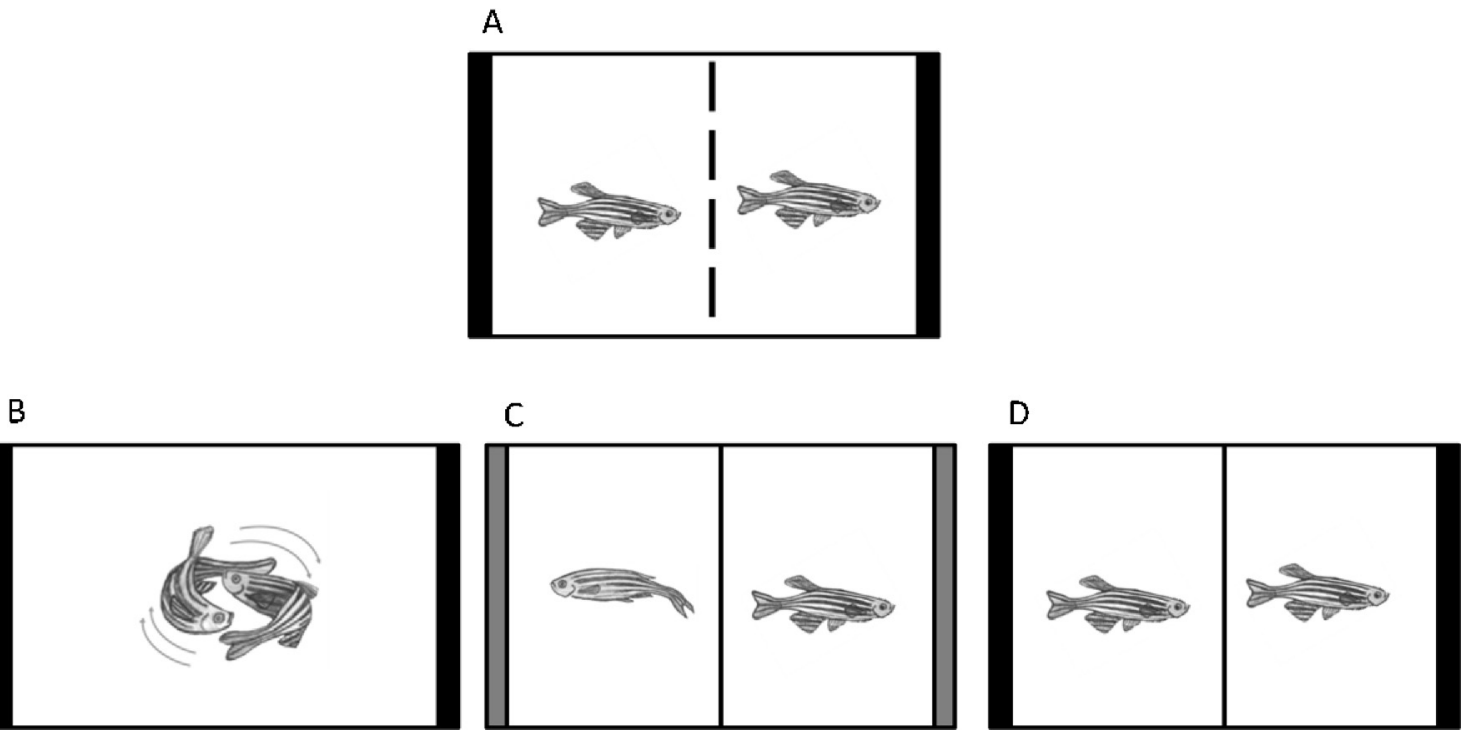

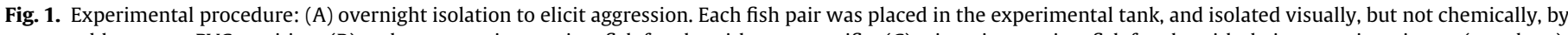

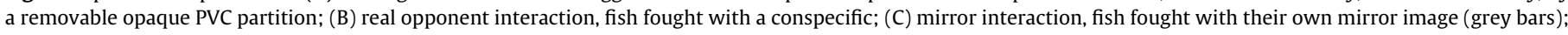
(D) control group, no agonistic interaction or mirror stimulation. 
Prior to the experiment, each pair was placed in the experimental tank $(20 \mathrm{~cm} \times 14.5 \mathrm{~cm} \times 12.5 \mathrm{~cm})$ where they were kept overnight in visual isolation using a removable opaque PVC partition. Previous studies had established periods of social isolation of 5 days [24] and $24 \mathrm{~h}$ [26] as effective to elicit aggressive behaviour. However, here we established that overnight isolation was sufficient to promote the consistent expression of aggressive behaviour. After the isolation period, the opaque divider was removed and the fish were allowed to interact for a period of $30 \mathrm{~min}$. Behavioural interactions were videotaped (JVC-Everio S Memory camcorder-GZMS215) for subsequent behavioural analysis (see below).

\subsection{Sampling}

In order to avoid monoamine degradation during the brain macro-dissection and to keep the time of sampling after the social interactions as homogeneous as possible across dyads, only one fish from each dyad was used for monoamine quantification. These fish were sacrificed immediately after the interaction with an overdose of tricaine solution (MS222, Pharmaq; $500-1000 \mathrm{mg} / \mathrm{L}$ ) and the spinal cord sectioned. The brain was macrodissected under a stereoscope (Zeiss; Stemi 2000) into five areas: Olfactory bulb and Telencephalon (OB/TL), Optic tectum (OT), Diencephalon (DE), Cerebellum (CB), and Brain stem (BS). Immediately after collection the brain tissue was placed on dry ice and stored at $-80{ }^{\circ} \mathrm{C}$ until analysis.

\subsection{Analysis of brain monoamines and metabolites}

The frozen macroareas were homogenized in $4 \%(\mathrm{w} / \mathrm{v})$ ice-cold perchloric acid containing $100 \mathrm{ng} / \mathrm{ml} \mathrm{3,4-dihydroxybenzylamine} \mathrm{(DHBA,} \mathrm{the} \mathrm{internal} \mathrm{standard)}$ using a Sonifier cell disruptor B-30 (Branson Ultrasonics, Danbury, CT, USA) and were immediately placed on dry ice. Subsequently, the homogenized samples were thawed and centrifuged at $21,000 \times \mathrm{g}$ for $10 \mathrm{~min}$ at $4{ }^{\circ} \mathrm{C}$. The supernatant was used for high performance liquid chromatography with electrochemical detection (HPLC-EC), analyzing the monoamines dopamine (DA) and serotonin (5-HT, 5-hydroxytryptamine) the DA metabolite DOPAC (3,4-dihydroxyphenylacetic acid) and the 5-HT metabolite 5-HIAA (5-hydroxy indole acetic acid), as described by Overli et al. [10]. In brief, the HPLC-EC system consisted of a solvent delivery as system model 582 (ESA, Bedford, MA, USA), an auto injector Midas type 830 (Spark Holland, Emmen, the Netherlands), a reverse phase column (Reprosil-Pur C18-AQ $3 \mu \mathrm{m}, 100 \mathrm{~mm} \times 4 \mathrm{~mm}$ column, Dr. Maisch HPLC GmbH, Ammerbuch-Entringen, Germany) kept at $40^{\circ} \mathrm{C}$ and an ESA 5200 Coulochem II EC detector (ESA, Bedford, MA, USA) with two electrodes at reducing and oxidizing potentials of $-40 \mathrm{mV}$ and $+320 \mathrm{mV}$. A guarding electrode with a potential of $+450 \mathrm{mV}$ was employed before the analytical electrodes to oxidize any contaminants. The mobile phase consisted of $75 \mathrm{mM}$ sodium phosphate, $1.4 \mathrm{mM}$ sodium octyl sulphate and $10 \mu \mathrm{M}$ EDTA in deionized water containing $7 \%$ acetonitrile brought to $\mathrm{pH} 3.1$ with phosphoric acid. Samples were quantified by comparison with standard solutions of known concentrations. To correct for recovery DHBA was used as an internal standard using HPLC software Clarity ${ }^{\mathrm{TM}}$ (DataApex Ltd., Prague, Czech Republic). The ratios of 5HIAA/5-HT and DOPAC/DA were calculated and used as an index of serotonergic and dopaminergic activity, respectively.

For normalization of brain monoamine levels, brain protein weights were determined with Bicinchoninic acid protein determination (Sigma-Aldrich, Sweden) according to the manufacturer's instructions. The assay was read on Labsystems multiskan 352 plate reader (Labsystems, Thermo Fisher Scientific) wavelength of $570 \mathrm{~nm}$.

\subsection{Behavioural observations}

Video recordings were analyzed using a computerized multi-event recorder (Observer XT, Noldus, Wageningen, The Netherlands). The zebrafish ethogram [26] was used as a reference and the observed behaviours were divided into aggressive (bite, chase and strike) and submissive (freeze and flee). As previously described in [26] dyadic male fights have two distinct phases: the pre-resolution phase where the fight is symmetric and both fish exhibit the same repertoire of behaviours (display, circle, and bite) and the post-resolution phase where all agonistic behaviours are initiated by the winner whereas the loser only displays submissive behaviours. Because we were only interested in the different output of the fights which generate different behavioural phenotypes (e.g. winner and loser) we only analyzed the post-resolution phase (i.e. the last $5 \mathrm{~min}$ of the $30 \mathrm{~min}$ interaction). We also measured the fight resolution time (time for the social hierarchy to be established) in order to compare real opponent with mirror interactions.

\subsection{Statistical analysis}

Statistical analyses were performed with the software STATISTICA v.10 (StatSoft, Inc., 2011). Parametric statistic was used given that the variables match the parametric parameters. One loser and one control were removed from the analysis, one because the output of its fight was not completely clear and the second because most of the time it was trapped on the partition, resulting in a sample size of 7 for losers and control groups, and 8 for winners and mirror groups. In the behaviour analyses, one animal from the winner, loser and mirror groups was removed from the analysis due to a problem with the video recordings which made the analysis impossible. A T-test was used to access differences between types of interactions (real opponent vs mirror) and fight resolution time. In the monoamines analysis four samples from the optic tectum were excluded due to problems during the sample preparation. Serotonin, dopamine levels and the respective metabolites, 5HIAA and DOPAC, as well as the activity of both neurotransmitters as measured by the ratios 5-HIAA/5-HT and DOPAC/DA, in brain macroareas were log transformed in order to meet the assumption of normal distribution. A repeated measures ANOVA (repeated factor: brain macroareas with 5 levels, independent factor: male status with 4 levels, winner, loser, mirror, control) was used to identify the main effects and the interaction between brain area and social status on the different monoamine measures, followed by a post hoc tests and planned comparisons of least squares means between the control group (isolation) and each of the different social status. A PCA analysis was used to reduce the number of behaviour variables in the real opponent paradigm. Correlations between behaviour and monoamine concentrations were obtained with Pearson correlation coefficients. All tests were two-tailed and statistical significance was set at $p<0.05$.

\subsection{Ethics statement}

The animal experimentation procedures used in this study followed the Association for the Study of Animal Behaviour and the Animal Behaviour Society guidelines for the treatment of animals in behavioural research and teaching and were approved by the internal Ethics Committee of the Gulbenkian Institute of Science and by the National Veterinary Authority (Direção Geral de Alimentação e Veterinária, Portugal; permit number 8954).

\section{Results}

\subsection{Behaviour}

In the real opponent paradigm all pairs except one, develop a clear dominant/subordinate relationship. Social hierarchies were stable and the behaviours exclusive for each phenotype. During the post-resolution phase a winner never became a loser nor a loser became a winner. The behaviours are stereotyped according to social status, aggressive behaviours in winners and submissive

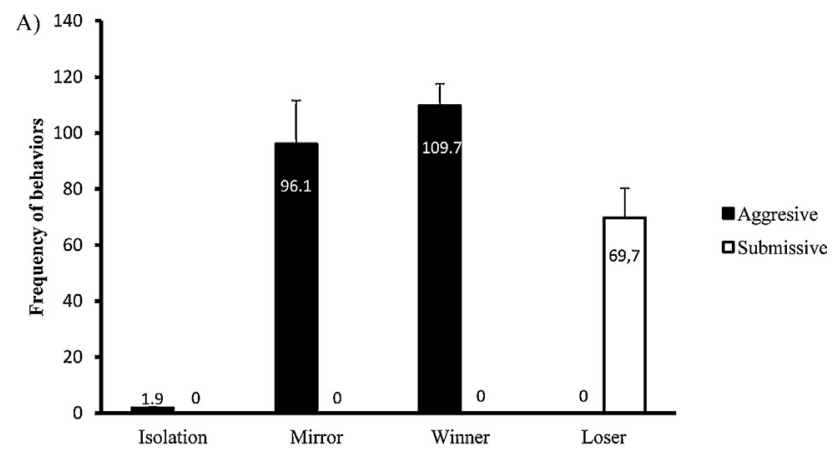

Figth resolution time

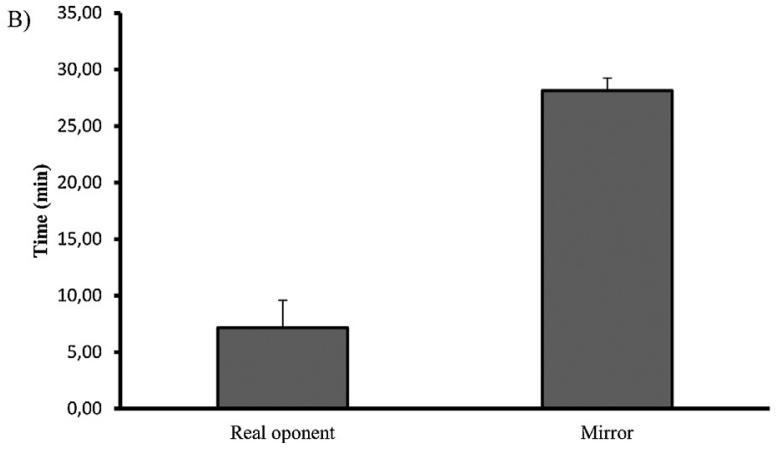

Fig. 2. Behavioural results. (A) Mean number of aggressive acts performed in the last $5 \mathrm{~min}$ of the $30 \mathrm{~min}$ agonistic interaction; error bars represent the standard error of the mean. (B) Fight resolution time, measured as the time needed for a social hierarchy to be established in the fighting male dyads (counting from the first bite to the post-resolution phase); error bars represent the standard error of the mean ( $t$-test: $T=-6.39, p<0.0001)$. 


\begin{tabular}{|c|c|c|}
\hline A) & PC1 & $\mathrm{PC} 2$ \\
\hline Bite & 0,9709 & $-0,0325$ \\
\hline Chase & 0,9405 & $-0,0077$ \\
\hline Freeze & $-0,52$ & $-0,8149$ \\
\hline Flee & $-0,7177$ & 0,4639 \\
\hline Strike & 0,8104 & $-0,06406$ \\
\hline Percent variation explained & $65,40 \%$ & $17,70 \%$ \\
\hline
\end{tabular}

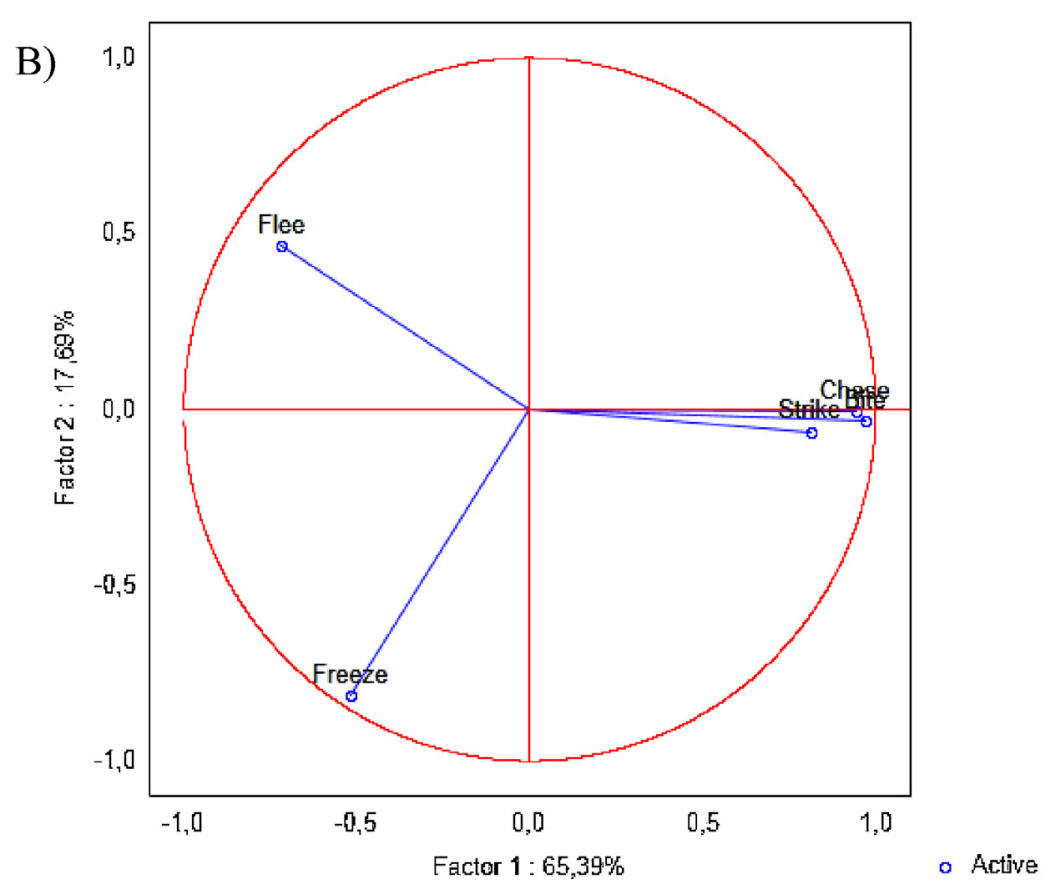

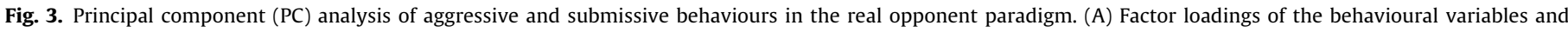

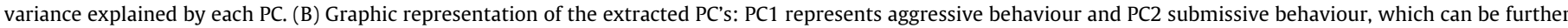
divided in active submission (flee) on the positive quadrant and passive (freeze) on the negative quadrant.

behaviours in losers. On the other hand, in mirror interactions because the fight is symmetric along time the resulting phenotype is not apparent, they never behave like losers or winners, and aggressive levels are kept constant during the whole interaction (Fig. 2). This difference is obvious in the fight resolution time $(T=-6.39, p<0.0001$; Fig. 2B) where mirror fighters fight for $30 \mathrm{~min}$ whereas in the real opponent interaction the fight is solved in approximately $7 \mathrm{~min}$, after which a post-resolution phase is established.

In order to reduce the number of behavioural variables in subsequent analyses in the real opponent paradigm, a Principal Component Analysis (PCA) was performed. Two factors, that together explain $83.1 \%$ of the total variance (Fig. 3A), were extracted that show a clear separation between aggressive and non-aggressive behaviours: PC1 has positive loadings for aggressive behaviours and a negative load for submissive behaviour (flee) and explains $65.4 \%$ of the variation; PC2 has positive loadings for submissive behaviour and negative loadings for all the aggressive behaviours (bite, chase, strike) and explains $17.7 \%$ of the variation (Fig. 3A). PC2 allows the subsequent division of submissive behaviour into an active (flee, positive quadrant) and a passive (freeze, negative quadrant) style (Fig. 3B). These results support the separation of aggressive and submissive behaviour in the real opponent interaction. In the mirror interaction, because the behavioural repertoire is restricted to two behaviours (bite, strike) no PCA was performed.

\subsection{Brain monoamines}

Concentrations of serotonin (5-HT), dopamine (DA) and their main metabolites (i.e. 5-HIAA and DOPAC, respectively) in the studied brain areas are given in Table 1.

There was a treatment and brain area main effect for both 5-HT (repeated measures ANOVA; social treatment: $F_{3,25}=7.86$ $p<0.001$; brain area: $F_{4,100}=79.39, p<0.0001$, respectively) and 5HIAA (repeated measures ANOVA; social treatment: $F_{3,24}=8.55$, $p<0.001$; brain area: $F_{4,96}=50.36, p<0.0001$ ). The post hoc analyses revealed that social experience increased 5-HT and 5-HIAA levels in animals that fought real opponents (W/L) and mirror image when compared to control group. For serotonin, the concentration was higher in the diencephalon, followed by olfactory bulb/telencephalon, optic tectum and brain stem and the lowest concentration was found in the cerebellum. On the other hand, for the metabolite 5-HIAA, olfactory bulb/telencephalon had the highest concentration, followed by diencephalon, optic tectum, brain stem and finally cerebellum.

For DA and DOPAC there was also a main effect for treatment and brain area. Social experience also increased DA $\left[F_{3,24}=5.03\right.$, 
Table 1

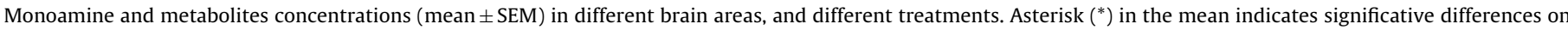
specific treatments when compared to control group (repeated measures ANOVA, ${ }^{*} p<0.05$ ).

\begin{tabular}{|c|c|c|c|c|c|c|}
\hline \multirow[t]{2}{*}{ Brain region } & \multirow{2}{*}{$\begin{array}{l}\text { Monoamines and } \\
\text { metabolites }\end{array}$} & \multicolumn{5}{|l|}{ Treatment } \\
\hline & & Control & Mirror fighter & Winner & Loser & Statistics \\
\hline \multirow[t]{4}{*}{ Telencepahlon } & 5-HT & $3.83 \pm 1.02$ & $6.40 \pm 0.85^{*}$ & $6.15 \pm 1.6$ & $5.61 \pm 0.74$ & $F(12,100)=2.48 ; p<0.01$ \\
\hline & 5-HIAA & $5.26 \pm 1.67$ & $9.06 \pm 1.66^{*}$ & $9.98 \pm 1.64^{*}$ & $9.05 \pm 1.28^{*}$ & $F(12,96)=1.17 ; p=0.32$ \\
\hline & DA & $2.08 \pm 0.57$ & $2.91 \pm 0.54$ & $2.57 \pm 0.53$ & $2.28 \pm 0.31$ & $F(12,96)=3.11 ; p<0.001$ \\
\hline & DOPAC & $0.46 \pm 0.16$ & $0.78 \pm 0.20$ & $0.68 \pm 0.08$ & $0.54 \pm 0.11$ & $F(12,100)=3.55 ; p<0.001$ \\
\hline \multirow[t]{4}{*}{ Diencephalon } & 5-HT & $8.86 \pm 1.43$ & $9.26 \pm 0.75$ & $8.19 \pm 0.95$ & $10.54 \pm 0.92$ & $F(12,100)=2.48 ; p<0.01$ \\
\hline & 5-HIAA & $4.44 \pm 0.71$ & $4.99 \pm 0.45$ & $4.22 \pm 0.33$ & $5.86 \pm 0.36^{*}$ & $F(12,96)=1.17 ; p=0.32$ \\
\hline & $\mathrm{DA}$ & $6.25 \pm 0.72$ & $6.23 \pm 0.52$ & $5.01 \pm 0.68$ & $6.93 \pm 0.65$ & $F(12,96)=3.11 ; p<0.001$ \\
\hline & DOPAC & $0.53 \pm 0.08$ & $0.67 \pm 0.06$ & $0.52 \pm 0.04$ & $0.83 \pm 0.09^{*}$ & $F(12,100)=3.55 ; p<0.001$ \\
\hline \multirow[t]{4}{*}{ Optic tectum } & 5-HT & $4.19 \pm 0.34$ & $4.11 \pm 0.36$ & $4.04 \pm 0.22$ & $3.55 \pm 0.28$ & $F(12,100)=2.48 ; p<0.01$ \\
\hline & 5-HIAA & $2.37 \pm 0.25$ & $2.11 \pm 0.39$ & $2.69 \pm 0.26$ & $2.87 \pm 0.26$ & $F(12,96)=1.17 ; p=0.32$ \\
\hline & DA & $0.92 \pm 0.07$ & $1.13 \pm 0.17$ & $1.01 \pm 0.06$ & $0.83 \pm 0.08$ & $F(12,96)=3.11 ; p<0.001$ \\
\hline & DOPAC & $0.18 \pm 0.01$ & $0.41 \pm 0.03^{*}$ & $0.28 \pm 0.02^{*}$ & $0.33 \pm 0.05^{*}$ & $F(12,100)=3.55 ; p<0.001$ \\
\hline \multirow[t]{4}{*}{ Cerebellum } & 5-HT & $0.32 \pm 0.06$ & $1.72 \pm 0.51^{*}$ & $1.47 \pm 0.25^{*}$ & $1.08 \pm 0.26^{*}$ & $F(12,100)=2.48 ; p<0.01$ \\
\hline & 5-HIAA & $0.92 \pm 0.49$ & $2.17 \pm 0.60^{*}$ & $1.47 \pm 0.60^{*}$ & $1.75 \pm 0.42^{*}$ & $F(12,96)=1.17 ; p=0.32$ \\
\hline & DA & $0.20 \pm 0.02$ & $1.63 \pm 0.63^{*}$ & $1.02 \pm 0.17^{*}$ & $0.72 \pm 0.17^{*}$ & $F(12,96)=3.11 ; p<0.001$ \\
\hline & DOPAC & $0.04 \pm 0.01$ & $0.30 \pm 0.09 *$ & $0.15 \pm 0.04^{*}$ & $0.18 \pm 0.05^{*}$ & $F(12,100)=3.55 ; p<0.001$ \\
\hline \multirow[t]{4}{*}{ Brain stem } & 5-HT & $3.62 \pm 0.56$ & $3.39 \pm 0.40$ & $6.51 \pm 1.18^{*}$ & $4.43 \pm 1.43$ & $F(12,100)=2.48 ; p<0.01$ \\
\hline & 5-HIAA & $2.37 \pm 0.32$ & $2.26 \pm 0.18$ & $3.15 \pm 0.36$ & $3.03 \pm 0.38$ & $F(12,96)=1.17 ; p=0.32$ \\
\hline & DA & $2.63 \pm 0.41$ & $2.23 \pm 0.21$ & $4.23 \pm 0.66^{*}$ & $3.21 \pm 0.86$ & $F(12,96)=3.11 ; p<0.001$ \\
\hline & DOPAC & $0.27 \pm 0.06$ & $0.35 \pm 0.03^{*}$ & $0.46 \pm 0.04^{*}$ & $0.41 \pm 0.08^{*}$ & $F(12,100)=3.55 ; p<0.001$ \\
\hline
\end{tabular}

$p<0.01)]$ and DOPAC levels $\left[F_{3,25}=8.31, p<0.001\right]$ in winners, losers and mirror fighters suggesting an activation of both systems in acute interactions. DA $\left[F_{4,96}=85.68, p<0.0001\right]$ distribution across the brain was distinct, with elevated concentrations in the diencephalon, then olfactory bulb/telencephalon and brain stem, and lastly optic tectum and cerebellum. For DOPAC $\left[F_{4,100}=39.09\right.$, $p<0.0001$ ] olfactory bulb/telencephalon and diencephalon exhibit the highest concentration, optic tectum and brain stem were after and cerebellum showed the lowest.

There was a significant main effect of brain area but not of social status in the ratios of both 5-HIAA/5-HT (repeated measures ANOVA, brain area main effect: $F_{4,88}=83.38, p<0.0001$; social status main effect: $F_{3,22}=1.27, p=0.31$ ) and DOPAC/DA (brain area main effect: $F_{4,68}=28.53, p<0.00001$; social status main effect: $F_{3,17}=2.17, p=0.13$ ). The post hoc analyses revealed that 5-HIAA/5-HT ratios were significantly higher in the olfactory bulb/telencephalon, followed by the cerebellum, then optic tectum and brain stem and lastly by the diencephalon. DOPAC/DA ratios were significantly higher in the optic tectum, followed by olfactory bulb/telencephalon, then cerebellum, and diencephalon and lastly in the brain stem. Contrast analysis of 5-HIAA/5-HT and DOPAC/DA activity of an area by area basis revealed that 5-HIAA/5-HT levels were significantly higher in winners' olfactory bulb/telencephalon $(F=18.43, p<0.001)$, and losers optic tectum $(F=9.92, p<0.01$; Fig. 4A). Regarding the DOPAC/DA, winners had higher activity levels in the olfactory bulb/telencephalon $(F=6.32, p<0.05)$, and mirror and losers in the optic tectum $(F=12.05, p<0.01$ and $F=6.67$, $p<0.05$ respectively; Fig. 4B). There was also a marginally nonsignificant tendency for losers to have increased DOPAC/DA ratios in the cerebellum $(F=3.96, p=0.06)$.

\subsection{Relationship between monoamines and behaviour}

Correlations analyses between behaviour and monoamine in different brain areas revealed that in the real opponent paradigm there were negative correlations between 5-HIAA levels $(r=-0.70$, $N=12, p<0.05)$ and DOPAC levels in the diencephalon $(r=-0.58$, $N=13, p<0.05$ ) and aggressive behaviour, and between 5HIAA/5HT ratio in the diencephalon and submissive behaviour $(r=-0.69$, $N=12, p<0.05$ ). Positive correlations were found between DA levels in the diencephalon and submissive behaviour $(r=0.60$, $N=13, p<0.05)$ and DA levels in the cerebellum and aggressive behaviour $(r=0.76, N=12, p<0.01)$.

In the mirror fighting treatment there were positive correlations between bite frequency and 5-HIAA levels in the optic tectum $(r=0.81, N=7, p<0.05)$, the 5HIAA/5HT ratios in the diencephalon $(r=0.90, N=7, p<0.01)$ and optic tectum $(r=0.83, N=7, p<0.05)$ and DOPAC/DA ratio in the diencephalon $(r=0.76, N=7, p<0.05)$. Strike frequency was negatively correlated with 5-HT and DOPAC
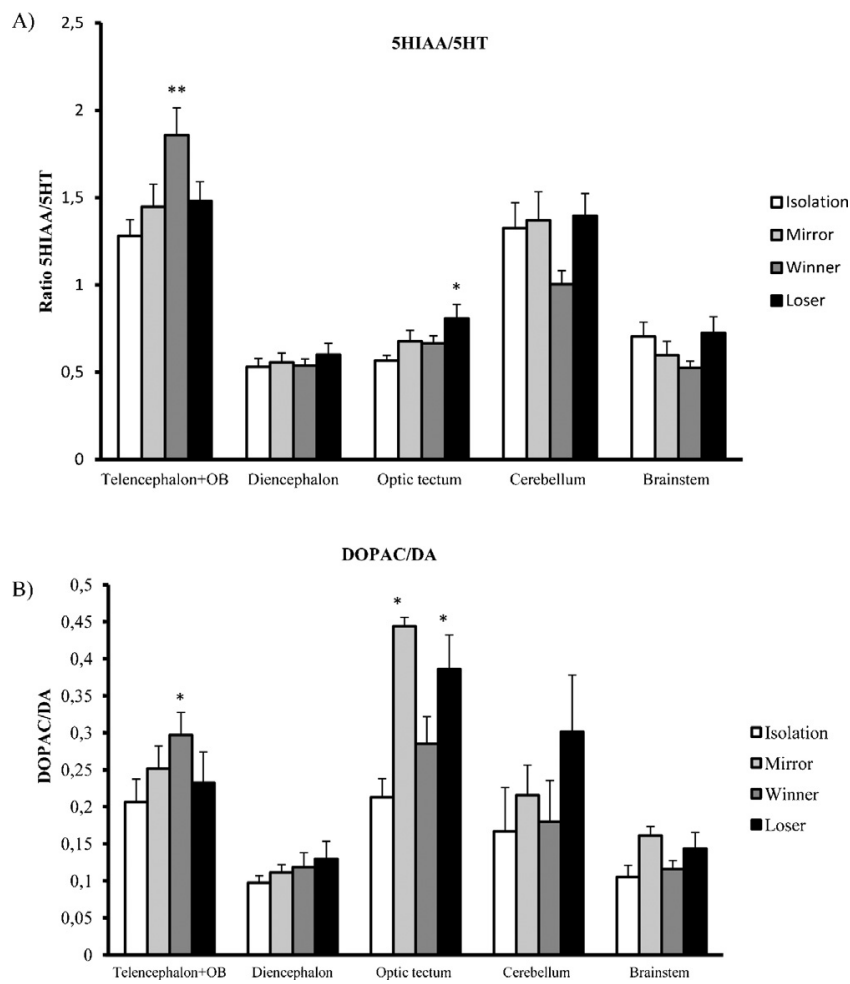

Fig. 4. Monoaminergic activity in different brain areas following an acute social interaction: (A) HIAA/5-HT ratio; (B) DOPAC/DA ratio. Error bars represent the standard error of the mean (repeated measures ANOVA, ${ }^{*} p<0.05$ and ${ }^{* *} p<0.01$ ). 
levels in the cerebellum $(r=-0.79, N=7, p<0.05 ; r=-0.79, N=7$, $p<0.05$ ) and positively correlated in the optic tectum with DA levels $(r=0.77, N=7, p<0.05)$. All other correlations were non significant.

\section{Discussion}

In the current study it is shown that following an acute agonistic encounter zebrafish males express two distinct behaviour profiles depending on the social status achieved: losers exhibit exclusively submissive behaviours, whereas winners express only aggressive behaviours (Fig. 2A). After the relative fighting ability has been established, the different behavioural repertoires for each social status are stable over time (at least up to 5 days, R.F. Oliveira and co-workers, unpublished data). For animals that fought their own mirror image only aggressive behaviours were observed, with a frequency that was not significantly different from that observed in winners of real opponent fights ( $T$-test: $T=-0.84$, $p=0.42$ ). However, a major difference between winners and mirror fighters is present, not on their behavioural output, but rather on the behaviour observed in the opponent, since in mirror fights the opponent (i.e. own image on the mirror) never displays submissive behaviours. As a consequence mirror fights were unsolved fights, as can be demonstrated by the fact that the expression of aggressive behaviour typical of the pre-resolution phase lasted for the whole duration of the trial (30 min), whereas in real opponent fights the encounter was resolved in approximately $7 \mathrm{~min}$ (after which post-resolution behavioural profiles were observed). Therefore, the experimental design used successfully produced four types of social phenotypes: winners, losers, individuals that expressed aggressive behaviour but did not experience either a win or a loss (i.e. mirror fighters), and individuals that did not express or perceived any social behaviour (control = social isolation). Therefore, the comparison of monoamine levels in regions of interest in the brain across these four social phenotypes allows the investigation of the shortterm effects of acute social interactions depending on perceived outcome by the participants.

For monoamines, we found that 5-HT levels are significantly higher in the telencephalon of mirror fighters, in the brain stem of winners and in the cerebellum of all experimental groups. The increase in 5-HT brain levels in the telencephalon and brain stem suggests that mirror fighters and winners are the groups where the serotonergic system is first activated in response to a social interaction and although they behave similarly, the brain areas activated are distinct which may indicate different perception of the context. We also found a brain area (i.e. cerebellum) that responds to acute stress independent of the interactions type (i.e. an increase in all groups was seen compared to controls).

For 5-HT metabolite (5-HIAA), significant increases were found in the telencephalon and in the cerebellum of all treatments (winner, losers, and mirror interaction), and in the diencephalon of losers. Interestingly, 5-HIAA levels in the diencephalon were negatively correlated with aggressive behaviour in the real opponent paradigm supporting the diencephalon enrolment in the regulation of aggressive behaviour. On the other hand, aggressive behaviour (bite frequency) was positively correlated with 5-HIAA in the optic tectum for mirror fighters. This later correlation may be primarily associated with increased visual stimulation in mirror fighters.

Our results suggest that acute interaction activated serotonergic system increasing 5-HT and 5-HIAA brain levels in response to different social conditions.

Serotonergic activity in turn, is significantly higher in the telencephalon of winners and in the optic tectum of losers, and no significant changes was observed in mirror fighters. Moreover, in real opponent fights serotonergic activity in the diencephalon was negatively correlated with submissive behaviour and in mirror fights serotonergic activity both in the diencephalon and in the optic tectum is positively correlated with overt aggression (i.e. bites). Given that social interaction did not affect 5-HT levels in these brain areas, 5-HT activity was mainly determined by metabolite levels. These results suggest that serotonergic activity is differentially regulated in different brain regions by social interactions. In zebrafish three clusters of serotonergic neurons have been described: the raphe nuclei, the posterior tuberculum/hypothalamic populations and the pretectal area. The telencephalon (including the olfactory bulbs) receives projections from the dorsal cells of the superior raphe $[27,28]$. Most of the 5-HT-ir fibres terminate in dorsolateral parts of the rostral telencephalon and a minor part continues ventrally into the olfactory bulb [29]. Thus, the observed increase in telencephalon and olfactory bulb serotonergic activity in winners may reflect an activation of the superior raphe projections in this social condition. Alternatively this increase in telencephalic serotonergic activity may be due to pre-synaptic stimulation of the terminal areas, which has been demonstrated, by disinhibition of GABAergic interneurons, increased glutamatergic local stimulation, and glucocorticoid infusion [30,31].

Most of the serotonergic fibres in the optic tectum seem to originate from serotonergic neurons of the pretectal cluster [29]. Pretectal nuclei, as well as the optic tectum, have been implicated in the regulation of visual and motor behaviour, multimodal sensory integration [32] and escape responses [33], which may explain the significant increased in subordinates or loser conditions, as observed in the present study. In mammals, avoidance responses are obtained from stimulations in a region of the superior colliculus that appears to represent the upper visual field [34]. Finally, serotonergic activity in the diencephalon which must represent the activation of the posterior tuberculum/hypothalamic 5-HT neuronal populations was positively correlated with overt aggression (i.e. bites) in the mirror fights and negatively correlated with submissive behaviour in real opponent fights, suggesting a role for these serotonergic populations in the balance between aggressive and submissive behaviour.

The activation of the serotonergic system in response to social interactions had been previously demonstrated for other species. In early stages of hierarchy formation the serotonergic system appears to be activated in both dominants and subordinates. For example, 5-HT levels were elevated after $10 \mathrm{~min}$ of social interaction in the limbic regions and in the locus coeruleus of dominant and subordinate fighting lizard males (in Anolis carolinensis) [35]. In rainbow trout (Oncorhynchus mykiss) both dominants and subordinates increased 5-HT activity in the telencephalon and optic tectum $3 \mathrm{~h}$ after the interaction [10]. Similarly, in the bicolor damselfish (Stegastes partitus), after a chronic interaction of $5 \mathrm{~d}$ dominants as well as subordinates showed higher levels of 5-HT activity in the telencephalon [36]. Other studies have shown that serotonergic activity has similar patterns in dominants and subordinates but this pattern seems to be temporally advanced in dominants [35]. Our data does not allow such comparison since we only collect one time point but we can speculate that the differences between social status in the brain are due to a time line that is acting at different speeds depending on social status, given that dominants and subordinates exhibit already differential patterns of 5-HT activation a short time after the resolution of the fight.

In the dopaminergic system there was a significant increase in DA levels in the cerebellum for all groups, and in the brain stem of winners. In the real opponent paradigm DA levels were positively correlated with aggressive behaviour in the cerebellum and in the diencephalon with submissive behaviour. For DOPAC, there was a significant increase for all groups in several brain areas; optic tectum, cerebellum and brain stem and in the diencephalon of losers. We also found a negative correlation of DOPAC in the diencephalon with aggressive behaviour. These results point out 
the contribution of diencephalon in the regulation of submissive behaviour. For mirror fighters DOPAC levels in the cerebellum were positively correlated with strikes.

On the other hand, dopaminergic activity was significantly higher in the telencephalon of winners and in the optic tectum of both losers and mirror fighters and these increases were mainly determined by the metabolite levels. Moreover, the expression of aggressive behaviour was positively correlated with dopaminergic activity in the diencephalon in mirror fights. Together these results suggest an involvement of the diencephalic monoaminergic system in the regulation of aggressive and submissive behaviours in different social conditions. This hypothesis is further supported by the known role of different diencephalic nuclei in the regulation of species-specific behaviours across vertebrates. For example, in the bluegill fish (Lepomis macrochirus) stimulation of the preoptic region inhibits aggressive behaviours and evoke courtship, and stimulation of a region surrounding the lateral recess elicits aggressive behaviour and feeding [37]. Similarly, in golden hamsters and rats, the anterior hypothalamus [38] and the nucleus accumbens [16] respectively, have been implicated in the regulation of aggressive behaviours, and in Syrian hamsters (Mesocricetus auratus) the nucleus accumbens is involved in conditioned defeat [39].

Dopamine release appear to be affected also in other brain areas, as the cerebellum and brain stem, but there were no significant differences in DOPAC/DA ratios since both the neurotransmitter and the metabolite levels increased in parallel indicating an increase in monoaminergic activity.

The increased dopaminergic activity in the telencephalon when males successfully achieve dominant status (i.e. winners) may be representative of social reward. A similar pattern has been previously observed in salmonids where dominant individuals showed higher DA activity in telencephalon than subordinate fish [40]. However, in contrast to amniotes, where the dopaminergic mesolimbic reward system is located in the ventral tegmental area (VTA), that project rostrally to the nucleus accumbens, amygdala and cortical areas (e.g. prefrontal cortex in mammals), fish do not present a midbrain dopaminergic population homologous to the VTA [41]. In contrasts, in fish the DA inputs to the telencephalon originate in a local subpallial DA system and in DA neurons in the ventral diencephalon, in particular in the posterior tuberculum, that project towards the subpallium [42-44]. Therefore, although evolutionary it cannot be considered as homologous to the mammalian VTA DA neurons, in fish this ascending DA pathway may be playing a similar role in reward behaviour as the mammalian mesostriatal DA pathway. On the other hand, the increased DA activity observed in losers and mirror fighters must be a consequence of the differential activation of another DA subsystem. A pretectal DA cell group (alar plate of p1) is consistently found in bony fishes, amphibians, and most amniotes except mammals [41]. These pretectal neurons are projecting mostly on the optic tectum, in a layer-specific fashion and they may play a role in the modulation of the retino-tectal visual input [45]. In this regard it is extremely interesting to note that the similar optic tetctum DA activation in mirror fighters and losers, despite the dissimilarities of their behavioural profile (i.e. mirror fighters are as aggressive as winners, and losers in contrast, are submissive), suggests that what is driving the DA activation in this region is the perception of the interaction, which is similar in mirror fighters and losers (i.e. both are exposed to an aggressive opponent), rather that the behavioural output of the focal individual.

In summary the data presented here confirms that acute social interactions elicit rapid and differential changes in serotonergic and dopaminergic activity across different brain regions in zebrafish. Further studies are needed to elucidate the specific roles of different neuromodulatory subsystem in the regulation of social behaviour. Finally, the ability of zebrafish reported here to respond to experimental manipulations of its social environment, combined with the fact that it is a species that expresses both gregarious (shoaling) and territorial behaviour, makes it a promising model organism in social neuroscience. In comparison to other established models in this field, such as cichlid fish (e.g. Astatotilapia burtoni [46]), zebrafish has the added value of having a large genetic tool box available that can be used to genetically dissect the mechanisms involved in social decision-making.

\section{Acknowledgements}

This study was funded by the Portuguese Foundation for Science and Technology (FCT, grants PTDC/PSI/71811/2006 and PEstOE/MAR/UI0331/2011 to RFO). During this study MT was being supported by a Ph.D. student fellowship by FCT(SFRH/BD/44848/2008). We thank to the two anonymous referees who contributed to the improvement of the final manuscript.

\section{References}

[1] Taborsky B, Oliveira RF. Social competence: an evolutionary approach. Trends Ecol Evol 2012;27:679-88

[2] Zupanc GKH, Lamprecht J. Towards a cellular understanding of motivation: structural reorganization and biochemical switching as key mechanisms of behavioral plasticity. Ethology 2000;46:467-77.

[3] Bargmann CI. Beyond the connectome: how neuromodulators shape neural circuits. Bioessays 2012;34:458-65.

[4] Libersat F, Pfluger HJ. Monoamines and the orchestration of behavior. Bioscience 2004; $54: 17-25$.

[5] Goodson JL, Thompson RR. Nonapeptide mechanisms of social cognition, behavior and species-specific social systems. Curr Opin Neurobiol 2010;20:784-94.

[6] Kravitz EA. Serotonin and aggression: insights gained from a lobster model system and speculations on the role of amine neurons in a complex behavior. J Comp Physiol A 2000;186:221-38.

[7] Huber R. Amines and motivated behaviors: a simpler systems approach to complex behavioral phenomena. J Comp Physiol A Neuroethol Sens Neural Behav Physiol 2005;191:231-9.

[8] Summers CH, Korzan WJ, Lukkes JL, Watt MJ, Forster GL, Overli O, et al. Does serotonin influence aggression? Comparing regional activity before and during social interaction. Physiol Biochem Zool 2005;78:679-94

[9] Winberg S, Nilsson GE. Roles of brain monoamines neurotrannsmitters in agonistic behaviour and stressreactions, with particular reference to fish. CompBiochemphysiol 1993;106:597-614.

[10] Overli O, Harris CA, Winberg S. Short-term effects of fights for social dominance and the establishment of dominant-subordinate relationships on brain monoamines and cortisol in rainbow trout. Brain Behav Evol 1999;54:263-75.

[11] Olivier B. Serotonin and aggression. Ann N Y Acad Sci 2004;1036:382-92.

[12] Dulawa SC, Gross C, Stark KL, Hen R, Geyer MA. Knockout mice reveal opposite roles for serotonin $1 \mathrm{~A}$ and $1 \mathrm{~B}$ receptors in prepulse inhibition. Neuropsychopharmacology 2000;22:650-9.

[13] Jenck F, Broekkamp CL, Van Delft AM. Effects of serotonin receptor antagonists on PAG stimulation induced aversion: different contributions of 5HT1, 5HT2 and 5HT3 receptors. Psychopharmacology (Berl) 1989;97:489-95.

[14] Sanchez C, Arnt J, Hyttel J, Moltzen EK. The role of serotonergic mechanisms in inhibition of isolation-induced aggression in male mice. Psychopharmacology (Berl) 1993;110:53-9.

[15] Sweidan S, Edinger H, Siegel A. D2 dopamine receptor-mediated mechanisms in the medial preoptic-anterior hypothalamus regulate effective defense behavior in the cat. Brain Res 1991;549:127-37.

[16] Van Erp AM, Miczek KA. Aggressive behavior, increased accumbal dopamine, and decreased cortical serotonin in rats. J Neurosci 2000;20:9320-5.

[17] Ferrari PF, van Erp AM, Tornatzky W, Miczek KA. Accumbal dopamine and serotonin in anticipation of the next aggressive episode in rats. Eur J Neurosci 2003; $17: 371-8$.

[18] Mos J, Van Valkenburg CF. Specific effect on social stess and aggression on regional dopamine metabolism in rat brain. Neurosci Lett 1979;15:325-7.

[19] Puglisi-Allegra S, Cabib S. Effects of defeat experiences on dopamine metabolism in different brain areas of the mouse. Aggress Behav 1990; 16:271-84.

[20] Louilot A, Le Moal M, Simon H. Differential reactivity of dopaminergic neurons in the nucleus accumbens in response to different behavioral situations. An in vivo voltammetric study in free moving rats. Brain Res 1986;397:395-400.

[21] Tidey JW, Miczek KA. Social defeat stress selectively alters mesocorticolimbic dopamine release: an in vivo microdialysis study. Brain Res 1996;721:140-9.

[22] Oliveira RF. Social plasticity in fish: integrating mechanisms and function. J Fish Biol 2012:81:2127-50.

[23] Spence R, Smith C. Male territoriality mediates density and sex ratio effects on oviposition in the zebrafish, Danio rerio. Anim Behav 2005;69:1317-23. 
[24] Larson ET, O‘Malley DM, Melloni Jr RH. Aggression and vasotocin are associated with dominant-subordinate relationships in zebrafish. Behav Brain Res 2006;167:94-102.

[25] Paull GC, Filby AL, Giddins HG, Coe TS, Hamilton PB, Tyler CR. Dominance hierarchies in zebrafish (Danio rerio) and their relationship with reproductive success. Zebrafish 2010;7:109-17.

[26] Oliveira RF, Silva JF, Simoes JM. Fighting zebrafish: characterization of aggressive behavior and winner-loser effects. Zebrafish 2011;8:73-81.

[27] Lillesaar C, Tannhauser B, Stigloher C, Kremmer E, Bally-Cuif L. The serotonergic phenotype is acquired by converging genetic mechanisms within the zebrafish central nervous system. Dev Dyn 2007:236:1072-84.

[28] Lillesaar C, Stigloher C, Tannhauser B, Wullimann MF, Bally-Cuif L. Axonal projections originating from raphe serotonergic neurons in the developing and adult zebrafish, Danio rerio, using transgenics to visualize raphe-specific pet1 expression. J Comp Neurol 2009;512:158-82.

[29] Kaslin J, Panula P. Comparative anatomy of the histaminergic and other aminergic systems in zebrafish (Danio rerio). J Comp Neurol 2001;440:342-77.

[30] Summers TR, Matter JM, McKay JM, Ronan PJ, Larson ET, Renner KJ, et al. Rapid glucocorticoid stimulation and GABAergic inhibition of hippocampal serotonergic response: in vivo dialysis in the lizard Anolis carolinensis. Horm Behav 2003;43:245-53.

[31] Barr JL, Forster GL. Serotonergic neurotransmission in the ventral hippocampus is enhanced by corticosterone and altered by chronic amphetamine treatment. Neuroscience 2011;182:105-14.

[32] Bally-Cuif L, Vernier P. Organization and physiology of the zebrafish nervous system. In: Perry SF, Ekker M, Farrell AP, Brauner CJ, editors. Zebrafish. Amsterdam: Elsevier; 2010.

[33] Herrero L, Rodriguez F, Salas C, Torres B. Tail and eye movements evoked by electrical microstimulation of the optic tectum in goldfish. Exp Brain Res 1998;120:291-305.

[34] Sahibzada N, Dean P, Redgrave P. Movements resembling orientation or avoidance elicited by electrical stimulation of the superior colliculus in rats. J Neurosci 1986;6:723-33.

[35] Summers CH, Summers TR, Moore MC, Korzan WJ, Woodley SK, Ronan PJ, et al. Temporal patterns of limbic monoamine and plasma corticosterone response during social stress. Neuroscience 2003;116:553-63.
[36] Winberg S, Myrberg Jr AA, Nilsson GE. Agonistic interactions affect brain serotonergic activity in an acanthopterygiian fish: the bicolor damselfish (Pomacentrus partitus). Brain Behav Evol 1996;48:213-20.

[37] Demski LS, Knigge KM. The telencephalon and hypothalamus of the bluegill (Lepomis macrochirus): evoked feeding, aggressive and reproductive behavior with representative frontal sections. J Comp Neurol 1971;143: $1-16$.

[38] Craig FF, Melloni Jr RH, Koppel G, Perry KW, Fuller RW, Delville Y. Vasopressin/serotonin interactions in the anterior hypothalamus control aggressive behavior in golden hamsters. J Neurosci 1997;17:4331-40.

[39] Luckett C, Norvelle A, Huhman K. The role of the nucleus accumbens in the acquisition and expression of conditioned defeat. Behav Brain Res 2012;227:208-14.

[40] Winberg S, Nilsson GE, Olsen KH. Social rank and brain levels of monoamines and monoamine metabolites in Arctic cbarr, SMvelinus Mpinus (L.). J Comp Physiol A 1991:241-6.

[41] Smeets WJ, Gonzalez A. Catecholamine systems in the brain of vertebrates: new perspectives through a comparative approach. Brain Res Brain Res Rev 2000;33:308-79.

[42] Rink E, Wullimann MF. The teleostean (zebrafish) dopaminergic system ascending to the subpallium (striatum) is located in the basal diencephalon (Posterior tuberculum). Brain Res 2001;889:316-30.

[43] Rink E, Wullimann MF. Development of the catecholaminergic system in the early zebrafish brain: an immunohistochemical study. Brain Res Dev Brain Res 2002:137:89-100.

[44] Tay TL, Ronneberger O, Ryu S, Nitschke R, Driever W. Comprehensive catecholaminergic projectome analysis reveals single-neuron integration of zebrafish ascending and descending dopaminergic systems. Nat Commun $2011 ; 2: 171$.

[45] Smeets WJ, Reiner A. Catecholamines in the CNS of vertebrates: current concepts of evolution and functional significance. In: Smeets WJAJ, Reiner A, editors. Phylogeny and development of catecholamine systems in the CNS of vertebrates. Cambridge: Cambridge University Press; 1994. p. $463-81$.

[46] Fernald RD. Social control of the brain. Annu Rev Neurosci 2012;35:133-51. 